\title{
Quranic Sign Language for Deaf People: Quranic Recitation Classification and Verification
}

\author{
Mohamed Ali Mahmod, Akram M. Zeki \\ Dept. of Information Systems, International Islamic University Malaysia, Kuala Lumpur, Malaysia \\ mmashadani@gmail.com \\ Dept. of Information Systems, International Islamic University Malaysia, Kuala Lumpur, Malaysia \\ akramzeki@iium.edu.my
}

\begin{abstract}
This paper provides an overview of the techniques used in image and video recognition for sign language through following hand motions and translating it to the text of the holy Quran. It also provides a proposal for a system that will be capable of identifying errors in Quran recitation depending on alphabets of Arabic and Quranic sign language and be able to show where exactly errors have occurred. In addition, this system will identify and classify location of verse (Ayah) and names of Souras depending on Back Propagation technique of the neural network.
\end{abstract}

Keywords- Arabic Quranic Sign Language, skin filtering, LDA, NN. Computer vision

\section{INTRODUCTION}

The recitation of the holy Quran must be free from mistakes. These mistakes may include; the missing verse or missing words at the same verse [1].

Memorizing the holy Quran and reviewing it continuously are the very important process to avoid forgetfulness. Sometimes, a person who memorizes and reviews his memorizing does not find everybody who can review his memorizing to him because of being busy or things like that.

Nowadays, many online applications contain Quranic recitation for different reciters, but it does not contain applications which enable hafiz (the person who memorizes and/or reviews the holy Quran) to review to him and virificate of his memorization.

This problem could be solved if there is a technique which will be able to highlight the errors online during the recitations process. There will be a need of maintaining the authenticity and the integrity of the recitation process of the holy Quran. We can note that there is no automated application that can recognize Quranic recitation using image and video recognition techniques.

Regarding this research, it will stress only on the Quranic recitation of image and video processing, which related to the holy Quran recitation. In addition, this proposed system offers deaf people (memorizers of the holy Quran) to recite the holy Quran through the sign language and then will be revised and corrected in realtime.

Furthermore, reading skills through sign language, deaf people can improve their reading skills during their recitation of the holy Quran depending on tracking hand motion.

\section{RELATED WORK}

Nowadays, many research was presented in the field of image and video processing for sign language and hand gesture recognition.

Al-Rousan et al [2] have used two neural network systems for the recognition of the sign language alphabets. They used Feed-Forward Neural Network and Probabilistic Neural Network for the purpose of classification.

By 2010, El-Bendary et al [3], used minimum distance classifier and multilayer perceptron classifier to improve the performance of an automated system to translate Arabic sign language into Arabic text.

Aliaa Youssif, et al [4], used Hidden Markov Model (HMM) for recognizing Arabic Sign language.

In 2012, Maraqa, et al [5] proposed using recurrent neural networks to recognize Arabic sign language. Naoum, et al [6] proposed a hybrid method of $K$ Nearest Neighbor (KNN) and Artificial Neural Network (ANN) to develop a hybrid system for Arabic sign language recognition.

Some studies used Eigen vectors and Eigen values weighted Euclidean distance for classification of sign language [7].

Mohandes et al [8] have compared the performance of Multilayer Perceptron neural networks with the Nave Bayes classifier in order to recognize Arabic sign language.

Tharwat. et al [9], have proposed the classifiers, Support Vector Machine (SVM), k-Nearest Neighbor (k-NN) to identify the Arabic sign characters.

A. Alphabet Character for The Arabic Language 
The Quranic Arabic alphabets consist of 28 letters, (from alif (')... until ya. Those letters include 25 letters, which represent consonants and 3 letters for vowels. The following figures 1and 2 illustrate Arabic characters and representing it in sign language.

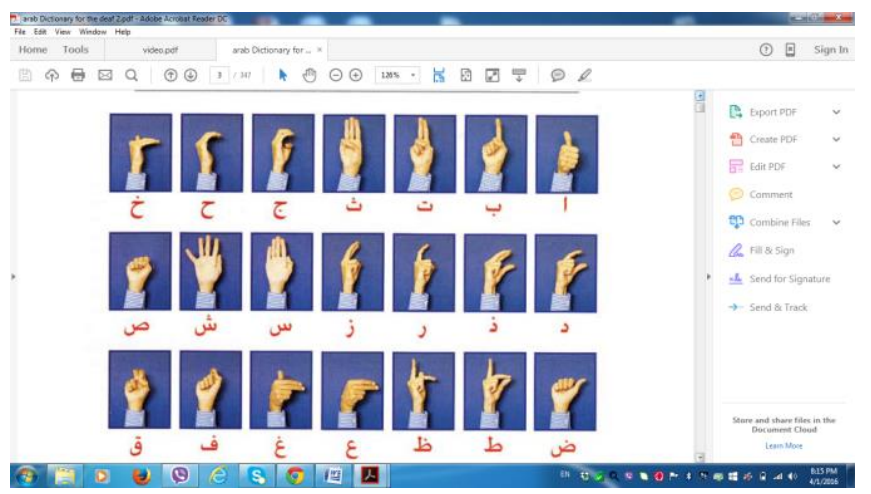

Figure 1: sign language representation for Arabic characters from (أ- ق)

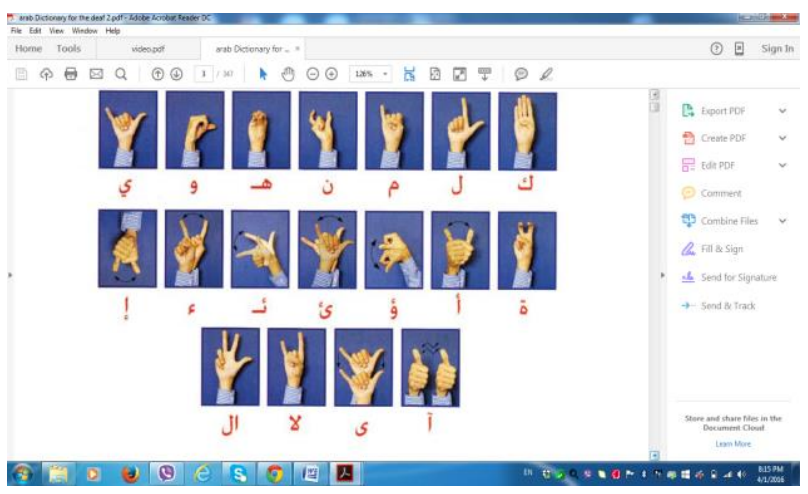

Figure 2: Sign language representation for Arabic characters for the remaining Arabic characters [10]

\section{IMAGE AND VIDEO RECOGNITION STAGES}

According to the existing studies, there are four main stages in recognizing images and videos. Under the same techniques of sign language (hand motion) recognition, the Quranic Arabic recitation recognition also can be implemented based on these techniques specified:

- Preprocessing.

- Feature extraction.

- Training/feature classification.

- Recognition/identification.

\section{Preprocessing}

Pre-processing has a crucial role in improving the performance of the Automatic sign language Detection process. It plays an important role in acquisition the data, hand gesture detection, and histogram matching and simplifying feature extraction process.
The main steps of pre-processing are:

\section{Data acquisition}

Data acquisition is considered the first step of preprocessing stage. It involves video capturing using webcam.

\section{Hand gestures detection}

This step in the preprocessing stage should involve using a specific filter for the purpose of skin detection.

Skin detection was achieved to the input video frames. This process is performed in order to separate skin colored regions from not skin colored regions.

\section{Histogram matching}

The next step after hand gesture detection is histogram matching which is used to describe the intensity of gradients of edges within specific shape for hand gesture detection [11].

\section{Feature Extraction AND CLASSIFICATION}

Feature Extraction process is so important to stage which could enable of extracting unique features for each shape of hand gesture. More specifically, the last frame is taken into consideration and vectors are extracted from this last frame.

There are different techniques which can be used to reduce dimension transforming the images to a subspace which is uncorrelated and to minimize the in-class scatter while maximizing the crossclass distance as shown as the following:

Principal Component Analysis (PCA).

Linear Discriminant Analysis (LDA) which is a method used in pattern recognition to find a linear combination of features which characterizes or separates two or more classes of objects or events. The resulting combination may be used as a linear classifier.

In our proposed system, we will focus on using LDA technique.

Moreover, there are some techniques that are used in classifying image and video processing for recognizing sign language as the following:

1) Support Vector Machine (SVM).

2) K-Nearest Neighbor (KNN).

3) Neural Network (NN).

In our proposed system, we will use and focus on the neural network technique.

Neural Network can be defined as Connection between the neurons in the different layers of the system. An example of the neural network consisted of three layers, the first layer has input neurons, which send data synapses to the second layer, and then more synapses to the third layer of the output neurons. More layers of neurons will make the 
system is more complicated. The neurotransmitters store parameters called "weights" that control the data in the calculations.

Artificial Neural Network can be depicted as the interconnection pattern between various layers of neurons. The learning procedure in a neural network is for updating the parameters "weights" of the interconnections. The enactment works that changes over a neuron's weighted input to its output activation. Figure 3 shows the three layers of the neural network composed of three layers: input, hidden and output.

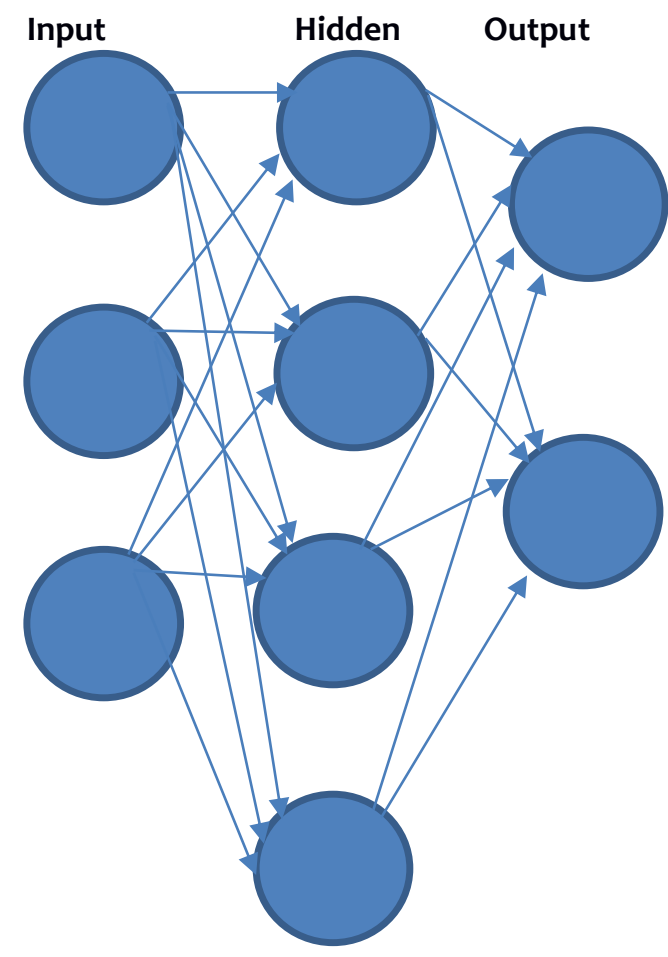

Figure3: three layers of a neural network

We will use the supervised learning of neural network technique called "backpropagation" which is still used because it is efficient for training the multilayer networks in image recognition process, which are capable in separating class regions of arbitrarily complicated distributions [12] [13]. In addition to that, the Backpropagation of Neural Network can be used also in video recognition and text recognition and other aspects of the artificial intelligence.

\section{THE PROPOSED SYSTEM}

The proposed system includes three major stages. The first one is the pre-processing stage which includes the skin filtering to detect skinned regions, hand gesture detection. To determine the similarity between frames, skin filtering and histogram matching histogram matching to determine the similarity between frames. The second and third stage is feature extraction and where the Eigen values and Eigen vector for LDA taken in consideration as features, where LDA techniques are to be used with Neural Network (NN) for classification of images and video of alphabets of sign language [14].

However, on the other hand, we will propose using backpropagation technique for the neural network for the purpose of verification of the recitation process of sign language through determining the location of the verse, name of the Soura and then missing verse and/or missing words in the same verse.

The following figures demonstrate the main work of this proposed system where Figure 4 shows an overview of the proposed system, while Figure 5 illustrates main steps for Automated Quranic recitation verification for deaf memorizers of the holy Quran.

The proposed system as illustrated in figure 4, starting with hand gesture motion detection which is the first step. The second step is the process of translating of the Hand Gesture (HG) to text. After that, the system will check and comparing with the correct training and saving data in the databases and check whether it is correct or incorrect, if it is correct, then the system will classify the Quranic verse (Aya) through comparing with the saved database of souras, verses names and ending the process. While if the text is incorrect, then the system will display the verification for the text and translating it to the Arabic Sign Language (ArSL) saved in the system and ending the process.

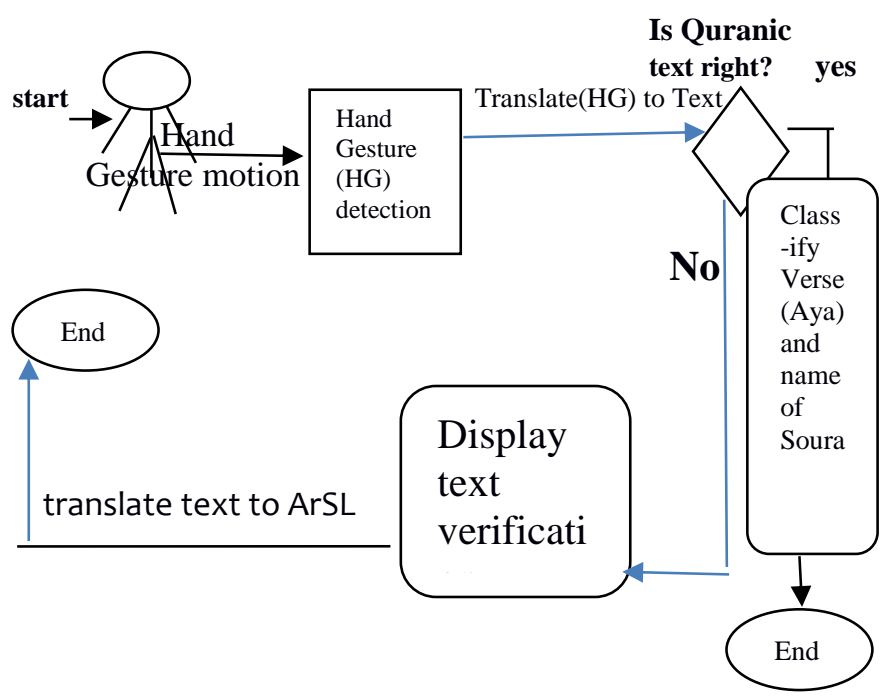

Figure 4: Overview of our proposed system

The main steps of the Automated Quranic recitation verification for deaf memorizers of the holy Quran can be summarized through inputting video, filtering of the skin 


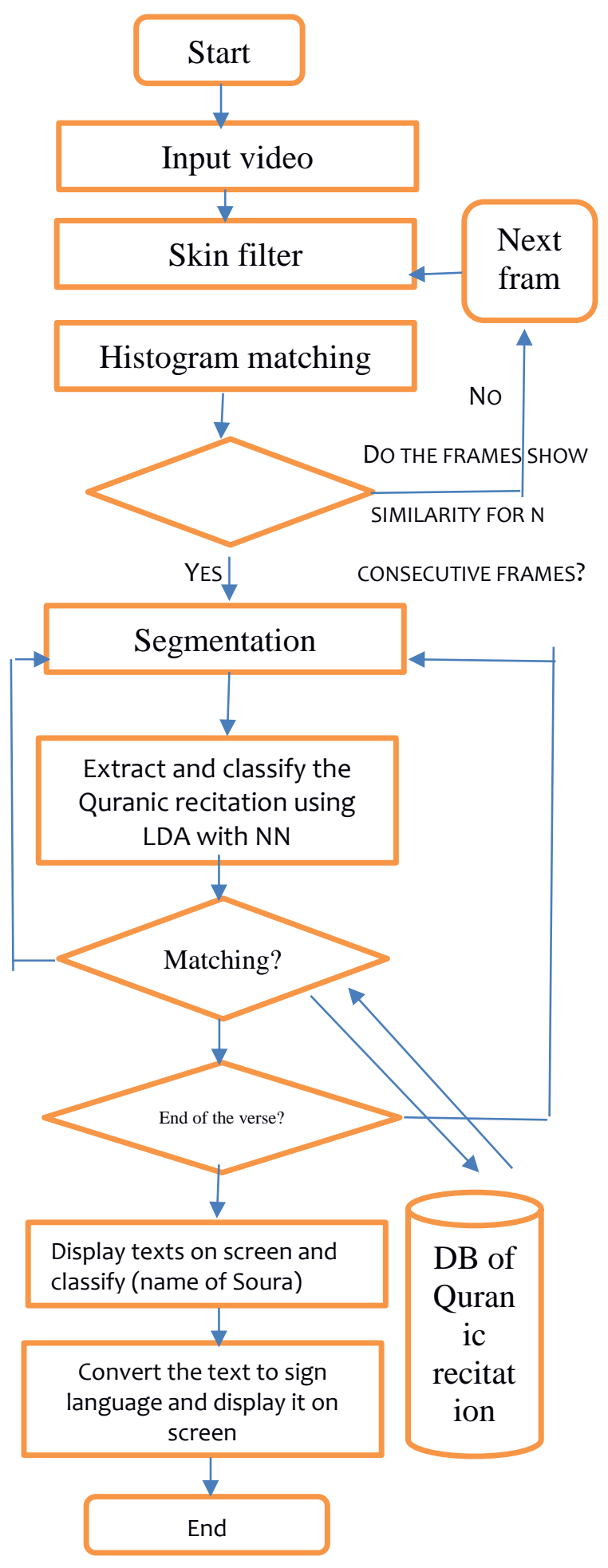

Figure 5: Automated Quranic recitation verification for deaf memorizers of the holy Quran. which it involves inputting image and converting Red Green Blue (RGB) coloured image to Grayscale image and histogram matching to check whether the frames show similarity for $n$ consecutive frames, if yes, then it goes to the segmentation process which decides the success or failure of any analysis for the process of edge detector. After that, the extraction of features and classification processes will take place using LDA and NN mentioned and explained above, and then matching process due to the database of the quranic verses will happen at the same time of training and testing process for LDA and NN. At this stage, the system will check the correction of the verses recited, if it correct, the system will display the text and classifies the Quranic verse (Aya) through comparing with the saved database of souras, verses names and ending the process. While if the text is incorrect, then the system will display the verification for the text and translating it to the Arabic Sign Language (ArSL) saved in the system and ending the process.

\section{CONCLUSION}

In this paper, we proposed a Quranic recitation system for deaf people through using alphabets of Arabic/Quranic sign language. The image and video recognition consist of three main stages: pre-processing composed of data acquisition, hand gesture detection, and histogram matching, while the second and third stages are feature extraction and classification. In this system, we propose using LDA technique with the $\mathrm{NN}$ technique for training and testing images and videos for alphabets of Arabic/Quranic Sign Language for video sequences. Furthermore, we propose using back propagation technique of neural network for training, testing, recognition and classification location of verse/Aya and name of Soura and verification of missing words or Aya for deaf people who memorize the holy Quran.

\section{REFERENCES}

[1] H. Tabbal, W. El-Falou, B. Monla, "Analysis and implementation of a "Quranic" verses delimitation system in audio files using speech recognition techniques". $2^{\text {nd }}$ International Conference on Information and Communication Technologies, Print ISBN 0-7803-9521- 2, Vol. 2, 2006, pp. 2979-2984.

[2] M. AL-Rousan, O. Al-Jarrah and N. Nayef, "Neural Networks Based Recognition System for Isolated Arabic Sign", 2006.

[3] N. Al-Bendary, H. M. Zawbaa ; M. S. Daoud ; A.E. Hassanien and K. Nakamatsu, "ArSLAT: Arabic Sign Language Alphabets Translator", International Journal of Computer Information Systems and Industrial Management Applications .ISSN 2150-7988 Volume 3, pp. 498-506, 2011.

[4] A. A.Youssif , A.E. Aboutabl, and H. H. Ali, "Arabic Sign Language (ArSL) Recognition System Using HMM", (IJACSA) International Journal of Advanced Computer Science and Applications ,Vol. 2, No. 11, 2011.

[5] M. Maraqa, F. Al-Zboun, M. Dhyabat and R. Abu Zitar, "Recognition of Arabic Sign Language (ArSL) Using Recurrent Neural Networks", 
Journal of Intelligent Learning Systems and Applications, vol. 4, pp. 41- 52, 2012.

[6] R. Naoum, H. H. Owaied and S. Joudeh, "Development of a New Arabic Sign Language Recognition Using K-Nearest Neighbor Algorithm", Journal of Emerging Trends in Computing and Information Sciences, ISSN 2079-8407, Vol. 3, no. 8, August 2012.

[7] J. Singha and K. Das, "Recognition of Indian Sign Language in Live Video", International Journal of Computer Applications (0975- 8887) Vol. 70- No.19, May 2013.

[8] M. Mohandes, S. Aliyu and M. Deriche, "Arabic sign language recognition using the leap motion controller", 2014.

[9] A. Tharwat, T. Gaber, A. E. Hassanien, M. K. Shahin and B. Refaat, "SIFT-Based Arabic Sign Language Recognition System", AfroEuropean Conference for Industrial Advancement vol. 334 of the series Advances in Intelligent Systems and Computing pp 359-370, retrieved on August 2017
[10] League of Arabic states, Arabic dictionary for deaf people, 2013.

[11] N. Dalal and B. Triggs, "Histograms of Oriented Gradients for Human Detection", IEEE Computer Society Conference on Computer Vision and Pattern Recognition (CVPR'05), ISSN 1063-6919, Vol 1. Pp. 886893, 2005.

[12] O. N. S. A. Boredi, "A hybrid ANN-SVM approach for Malay word check recognition system", Master thesis, 2012

[13] H. K. Mohair, J. M. Saleh, and S. A. Suandi, “ Human skin color detection: a review on neural network perspective, International Journal of Innovative Computing, Information, Control, Vol. 8, no. 12, pp. 8115-8131, 2012.

[14] J. Singha and K. Das, "Recognition of Indian sign language in Live video", International Journal of Computer, vol. 70, no. 19, pp. 975-8887, 2013 